\title{
The Implications of Pricing on Social Learning.
}

\author{
ITAI ARIELI, MORAN KOREN, and RANN SMORODINSKY, Technion - Israel Institute of \\ Technology.
}

In many markets of substitute products, the value of the various alternatives may depend on some unknown variable. This may take the form of some future change in regulation, a technological shock, environmental developments, or prices in related upstream markets. Although this information is unknown, individual consumers may receive some private information about these fundamentals. We ask whether, in such an environment, markets aggregate information correctly and the ex-post superior product eventually dominates the market.

In this work we focus on the role of social learning in such environments. We study whether the learning process guarantees an efficient outcome. We isolate the role of learning by introducing a simple duopoly model of common value in which consumers, with a unit demand, choose between two substitute products, each with zero marginal cost of production. The timing of the interaction is as follows. Nature randomly chooses one of two states of nature, and so determines the identity of the firm with the superior product. At each stage the two firms observe the entire history of the market - past prices and consumption decisions and simultaneously set prices. Thereafter, a single consumer arrives and receives a private signal regarding the state of nature. The consumer makes his consumption decision based on his signal, the pair of prices for each product, and the entire history of prices and consumption decisions. The consumer can also choose to opt out of buying any product. Our main goal is to identify conditions under which asymptotic learning holds; that is, information is fully aggregated in the market asymptotically.

When prices are set exogenously and fixed throughout, the above model is exactly the standard herding model [1, 2]. In that model, as shown by Smith and Sørensen [3], the characterization of asymptotic learning crucially depends upon the quality of agents' private signals. In particular, one must distinguish between two families of signals: bounded versus unbounded. In the unbounded case the private beliefs of the agents are, with positive probability, arbitrarily close to zero and one. Therefore, no matter how many people herd on one of the alternatives, there is always a positive probability that the next agent will receive a signal that will make him break away from the herd toward the other alternative. This property, as shown by Smith and Sørensen [3], entails asymptotic learning. The same logic applies in our model as well: when signals are unbounded, even if the prior is extremely in favor of one product, with positive probability there will be a consumer who gets a sufficiently strong signal,that tilts the consumption decision toward the a priori inferior product; thus, under strategic pricing and unbounded signals, asymptotic learning holds.

The learning results in our model depart from those of the canonical model when signals are bounded. In the herding model there is always a positive probability that the suboptimal alternative will eventually be chosen by all agents. However, intuition suggests that when prices are endogenized they serve to prevent such a herding phenomenon. Hypothetically, once society stops learning and a herd develops on the product of one firm, the other firm will lower its product price to attract new consumers and learning will not cease. It turns out that this intuition, although not entirely correct, does have some merit. In order for the intuitive argument to hold, signals must exhibit a property referred to here as vanishing likelihood.

When signals are bounded the posterior belief of any agent, given his signal, is bounded away from zero and one for any interior prior. The proportion of agents whose posterior lies within $\varepsilon$ of the posterior distribution's

Permission to make digital or hard copies of part or all of this work for personal or classroom use is granted without fee provided that copies are not made or distributed for profit or commercial advantage and that copies bear this notice and the full citation on the first page. Copyrights for third-party components of this work must be honored. For all other uses, contact the owner/author(s).

EC '19, June 24-28, 2019, Phoenix, AZ, USA

(C) 2019 Copyright held by the owner/author(s).

ACM ISBN 978-1-4503-6792-9/19/06.

https://doi.org/10.1145/3328526.3329554 
boundaries obviously shrinks to zero as $\varepsilon$ goes to zero. We say that signals exhibit vanishing likelihood if the density of consumers at the posterior belief distribution's boundaries goes to zero.

Consumers who receive signals that induce such extreme posterior beliefs are those consumers who are likely to go against a herd and purchase the less popular product. We refer to such consumers as nonconformists. With this interpretation in mind the property of "vanishing likelihood" serves as a measure of the prevalence of nonconformism. More particularly, we associate vanishing likelihood with a negligible level of nonconformism while signals that do not exhibit vanishing likelihood are associated with significant nonconformism.

When society herds, each agent follows in the footsteps of his predecessors and thus, intuitively, one expects nonconformism (when signals do not exhibit vanishing likelihood) to induce learning. Our main result shows that the opposite occurs: in the presence of strategic pricing asymptotic learning holds if and only if signals have the vanishing likelihood property.

The full paper can be found at http://bit.ly/ec19aks.

CCS Concepts: • Information systems $\rightarrow$ Electronic commerce; • Theory of computation $\rightarrow$ Algorithmic game theory and mechanism design; Algorithmic game theory; Convergence and learning in games; Market equilibria; • Computing methodologies $\rightarrow$ Sequential decision making; Markov decision processes; $\bullet$ Applied computing $\rightarrow$ Decision analysis; Marketing.

Additional Key Words and Phrases: game theory, social learning, pricing, dynamic, stochastic, information aggregation, strategic behavior, innovation,disruption

\section{ACM Reference Format:}

Itai Arieli, Moran Koren, and Rann Smorodinsky. 2019. The Implications of Pricing on Social Learning.. In ACM EC '19: ACM Conference on Economics and Computation (EC '19), June 24-28, 2019, Phoenix, AZ, USA. ACM, New York, NY, USA, 2 pages. https://doi.org/10.1145/3328526.3329554

\section{ACKNOWLEDGMENTS}

Arieli acknowledges the support of the Ministry of Science and Technology (grant 19400214), and the Bernard M.Gordon Center for System Engineering at the Technion (grant number 43100038 ).

Smorodinsky gratefully acknowledges United States-Israel Binational Science Foundation and National Science Foundation grant 2016734, the German-Israel Foundation grant I-1419-118.4/2017, the Ministry of Science and Technology grant 19400214, Technion VPR grants, and the Bernard M. Gordon Center for Systems Engineering at the Technion.

\section{REFERENCES}

[1] Av Banerjee. 1992. A Simple Model of Herd Behavior. The Quarterly fournal of Economics 107, 3 (1992), $797-817$. https://doi.org/10.2307/2118364

[2] Sushil Bikhchandani, David Hirshleifer, and Ivo Welch. 1992. A Theory of Fads, Fashion, Custom, and Cultural Change as Informational Cascades. Fournal of Political Economy 100, 5 (1992), 992-1026. https://doi.org/10.1086/261849

[3] B Y Lones Smith and Peter Sørensen. 2000. Pathological Outcomes of Observational Learning. Econometrica 68, 2 (2000), 371-398. 